\title{
Cerebellar Involvement in Clumsiness and Other Developmental Disorders
}

\author{
Richard B. Ivry \\ Department of Psychology, University of California, Berkeley, California, USA
}

\begin{abstract}
Cerebellar abnormalities have been linked to a number of developmental disorders. Much evidence is based on the analysis of highresolution MRI scans. Imaging and behavioral studies have led researchers to consider functional contributions of the cerebellum beyond that associated with motor control. I review this literature, providing an analysis of different ways to consider the relation between cerebellar abnormalities and developmental disorders. Interestingly, although clumsiness is a problem of coordination, the contribution of cerebellar dysfunction to this developmental problem has received little attention. Select studies indicate that some clumsy children have difficulties on tasks requiring precise timing, similar to that observed in adult patients with cerebellar lesions. I suggest that the underlying neural bases of clumsiness are heterogeneous, with cerebellar dysfunction likely a major contributor for a subpopulation of such children.
\end{abstract}

\section{INTRODUCTION}

Problems with motor coordination are a common feature of neurologic disorders, consistent with the observation that a substantial proportion

Reprint requests to: Richard B. Ivry, Department of Psychology, MC 1650, University of California, Berkeley, California 94720, USA; e-mail: ivry@socrates.berkeley.edu of the central nervous system is associated with the control of movement. Parkinson's disease and Huntington's disease are examples of degenerative disorders of the brain having prominent motor disturbances. Syndromes like hemiplegia or apraxia are often present following stroke, although persistence is dependent on the extent and location of the resultant neuropathology.

Developmental disorders affecting coordination have received modest attention in the cognitive neuroscience literature. This situation likely reflects many factors, such as difficulty in defining appropriate populations, unique laboratory demands involved in testing children, and that coordination problems, at least for some children, become less pronounced with maturation. For example, many children who exhibit delayed development in reading eventually catch up with their peers as young adults, or at least acquire a sufficient skill level so that the problem does not interfere with their education or careers (Demb et al., 1998).

The idea that certain developmental disorders can be linked to specific neurologic abnormalities has only recently taken firm hold in the neuroscience community. This paradigm shift is driven not only by new methodologies for analyzing brain function, but also by the application of sophisticated behavioral tests for assaying cognitive and motor abilities. Rather than focusing on standardized tests that provide useful descriptions of performance, the methods of cognitive psychology are designed to isolate the set of specific mental operations that are invoked in the performance of complex skills. Whether this new approach will 
prove fruitful in the study of clumsiness remains to be seen.

The term 'clumsiness' describes a rather broad set of behaviors. Establishing a defining set of criteria has been difficult and the subject of much debate (Henderson \& Henderson, 2003). The general consensus is that this label refers to a heterogeneous constellation of coordination problems. Such heterogeneity can be viewed in at least two different ways. One interpretation is that the diversity arises from diffuse neurologic abnormalities. Alternatively, heterogeneity might reflect the use of a term in a generic way, even though subtypes exist that result from more focal neural dysfunction.

This paper focuses on the relation of cerebellar function to clumsiness. The cerebellum is a prime structure to consider when discussing the role of specific neural systems in coordination problems. The most prominent symptom observed in patients with acquired cerebellar disorders is a loss of coordination. Similar to the behavior of clumsy children, such patients generally have a good sense of the appropriate action for a given context; their problems arise when trying to execute the movements in a coordinated manner. The term 'cerebellar ataxia' is used to describe the breakdown in patterns of muscular activation that cause the limb to follow a wobbly trajectory or fail to end at a target location.

Another reason to consider the relation between the cerebellum and clumsiness comes from the recent association of this structure to a number of disparate developmental disorders. This association has led to new perspectives on cerebellar function-perspectives that emphasize non-motor capabilities (see Schmahmann \& Harris, 1997). The first section of this paper provides a brief review of recent literature, addressing the issue of how we should interpret these correlations. Following this, I explore the more specific question of whether cerebellar dysfunction is apparent in clumsy children, and whether such deficits are ubiquitous in this population or restricted to subgroups.

\section{CEREBELLAR ASSOCIATION WITH DEVELOPMENTAL DISORDERS}

Evaluating causal brain-function relationships is a tricky business. Neuropsychological research is primarily correlational. In the best-case scenario, functional inferences are made about neural structures based on the observation that consistent behavioral impairments arise following neuropathology restricted to a well-localized region. In more typical situations, the damage spans relatively large areas. Moreover, it remains possible that the behavioral changes are due to indirect alterations in the function of intact tissue. With developmental disorders, the challenge is even greater. Neural abnormalities can be subtle and/or relatively diffuse. In addition, as discussed in various papers in this special edition, there are high degrees of co-morbidity of many syndromes. For example, clumsiness has been reported to be more prevalent in both children with attention deficit and hyperactivity disorder (ADHD) and dyslexia.

When considering the relation of the cerebellum to clumsiness, it is instructive to examine other developmental disorders that have been linked to this structure. Much of this work is based on neuroanatomic analyses made possible with magnetic resonance imaging (MRI). This technique allows for the in vivo analysis of brain structure with remarkable spatial resolution. Measurements are made to determine if particular disorders are associated with structural abnormalities, usually in terms of volumetric deviations Although the application of this technique using large samples of clumsy children is just emerging (Mercuri \& Barnett, 2003), MRI has been used to 
study a number of psychiatric and developmental disorders. A striking result in this literature has been the surprising degree of cerebellar pathology observed in disorders that would seem, a priori, to have little connection to cerebellar function.

Perhaps best studied with this approach is autism. In 1988, Courchesne and colleagues (1988) reported pronounced cerebellar hypoplasia in a study that included 18 autistic individuals and 12 age-matched controls (mean age $=20.9$ years ranging from 6 to 30 years old). Interestingly, no other brain region showed a difference between the two groups. Subsequent studies involving larger sample sizes have confirmed that cerebellar abnormalities are consistently associated with autism, although the initial report has been qualified in two significant ways. First, a subpopulation of autistic individuals was found to have cerebellar hyperplasia (Courchesne et al., 1994). Second, the structural differences are not restricted to the cerebellum. Several MRI studies have shown reduced volume in the parietal lobe, limbic regions, and white matter tracts such as the corpus callosum (reviewed in Courchesne, 1997). Although the latter results suggest diffuse developmental abnormalities, apparently a reduced cerebellar volume is the most consistent structural marker of autism, at least in terms of macroscopic measurements of the central nervous system.

Cerebellar hypoplasia has been associated with two other psychiatric conditions, ADHD (Berquin et al., 1998; Mostofsky et al., 1998) and schizophrenia (Nopoulos et al., 1999). Even more so than with autism studies, MRI evidence with ADHD and schizophrenia indicates that cerebellar abnormalities co-exist with structural differences in the cerebral cortex. For example, children with ADHD show approximately $10 \%$ reduction in surface area of cerebellar lobules VIII to $\mathrm{X}$ and a $10 \%$ reduction in total volume of the cerebrum (Berquin et al., 1998). Although the last point emphasizes that the anatomic abnormalities are not restricted to the cerebellum, noteworthy is that the evidence to date argues for some degree of specificity. The regions within the cerebellar cortex showing a significant degree of hypoplasia differ for autism, ADHD, schizophrenia (see Fig. 1). Indeed, when standard divisions of the cerebellar cortex are used, the abnormalities associated with these syndromes form non-overlapping groups. This result argues against the idea that the cerebellum is generically sensitive to some sort of neural insult during development. A reasonable alternative is that the time course of neural development within these regions varies in a systematic manner (Altman \& Bayer, 1985), and that pathology-inducing events (genetic or environmental) have time sensitive windows of opportunity.

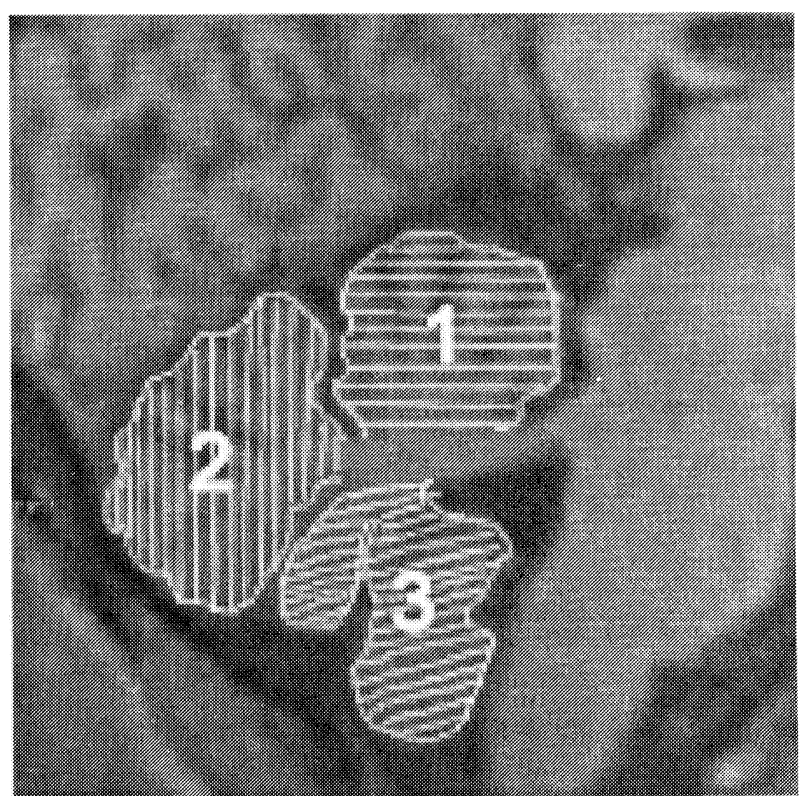

Fig. 1: Sagittal section of the cerebellum showing the folia of the vermis. MRI studies have revealed cerebellar hypoplasia associated with autism, schizophrenia, and ADHD. Interestingly, the regions showing hypoplasia differ for these disorders with the focus being in (1) Lobules I to $\mathrm{V}$ in schizophrenia; (2) Lobules VI to VII in autism; (3) Lobules VIII to X in ADHD. 
Researchers have also used behavioral methods to study the relation of cerebellar function to develop-mental disorders. One notable example is the recent work on developmental dyslexia. Children with severe reading problems have marked impairments on tests of coordination, and their problems resemble those exhibited by neurology patients with acquired cerebellar lesions (Fawcett et al., 1996). Moreover, on various motor and non-motor tests specifically designed to evaluate cerebellar function, dyslexic children perform in a manner similar to that of patients with cerebellar insults (Fawcett \& Nicolson, 1999; Nicolson et al., 2001).

To summarize this brief review, cerebellar abnormalities, either anatomically or behaviorally defined, have now been linked to developmental disorders like autism, ADHD, and dyslexia. The results are surprising in that motor problems have not been traditionally associated with any of these syndromes. As with all correlational results, we must give careful thought to our interpretation of these relations, how we assess potential cause-andeffect relations.

At one extreme, the correlations between cerebellar pathology and these developmental disorders may have no causative relation. For example, problems in development that can result in autism might independently produce hypoplasia of cerebellar lobules VI and VII. Or the underlying mechanisms could be very different but covary.

Alternatively, there may be causal relations between the cerebellar abnormalities and some or all of these developmental disorders. Over the past 10 years, well-articulated hypotheses have been offered about how cerebellar pathology could be central to the development of autism (Courchesne \& Allen, 1997), schizophrenia (Wiser et al., 1998), and dyslexia (Nicolson et al., 2001). These hypotheses build on more traditional notions concerning how the cerebellum might contribute to motor control. Andreassen et al. (1996) coined the term 'cognitive dysmetria' to describe the breakdown of thought patterns in schizophrenia. In this view, the cerebellum coordinates mental activity across regions of the cerebral cortex, similar to how it has been hypothesized to coordinate activity across different muscular groups for skilled movement. Courchesne and Allen (1997) proposed a more specific version of this idea with respect to a causal account of the cerebellum and autism. In his theory, the cerebellum is responsible for coordinating rapid shifts of attention. An inability to engage in coordinated attentional focus is seen as a fundamental deficit in the development of normal social relationships. With respect to dyslexia, Nicolson and Fawcett (2001) hypothesize that reading is one form of a skilled behavior, and that the cerebellum is essential for the automatization of skills.

At present, such causal accounts are speculative, yet can be subjected to rigorous empirical evaluation. The devil is in the details and the hypotheses will surely become more explicit (and thus testable) as terms like mental coordination or automatization become operationalized. Traditional neurology would encourage skepticism with respect to accounts in which cerebellar pathology is causally related to such disparate disorders such as autism, ADHD, and dyslexia.

- First, there are marked differences between these developmental disorders and it is not obvious why they would be related to a common neural system.

- Second, these theories tend to focus on the idea that a fundamental and localized pathology underlies the syndromes. Although this position may be useful for challenging traditional views, it is also likely to be simplistic.

- Third, patients with acquired cerebellar disorders do not appear to develop problems similar to those evident in autism, ADHD, or dyslexia. Patients with focal, unilateral lesions or widespread bilateral cerebellar degeneration 
do not have pronounced, if indeed, any deficits in coordinating rapid shifts of attention (Helmuth et al., 1997; but see Townsend et al., 1999).

The last point, though, must be qualified. Disturbing a system during development could have very different long-term consequences than would a similar disruption in a mature system. The study of infants who incur severe brain injuries has shown that massive functional reorganization is possible. The degree of recovery in such infants far exceeds that possible in adults who suffer similar injuries.

On the other hand, the dysfunctional operation of a system early in life can prevent the development of certain abilities, whereas those abilities can remain undisturbed if the same system is damaged late in life. Consider one of the hypotheses proposed to account for the putative relationship between cerebellar dysfunction and dyslexia (Ivry et al., 2001). In this hypothesis, the cerebellum is conceptualized to be part of an internal articulatory loop, contributing to covert articulation in a manner similar to how it participates in overt articulation. Building on the idea that our phonological knowledge develops by reference to the motor events that produce these sounds, one would expect that articulatory skill is essential for developing robust phonological representations. If cerebellar pathology disrupts the articulatory system, then normal development of phonological skills might be impacted. Learning to read would prove challenging, given the need to learn the mapping between orthography and phonology (Studdert-Kennedy \& Mody, 1995). Damage to the cerebellum in the adult, however, might have no effect on reading skills. With such individuals, the mapping between orthography and phonology should be well established. Moreover, skilled readers can directly access lexical representations from orthography without mediation through phonology (e.g., Coltheart et al., 2001).
This last hypothesis emphasizes one additional important issue concerning causal models of brain function and behavior. Causality can vary in terms of the degree of directness. In the hypothesis just discussed, the causal relation between the cerebellum and dyslexia is indirect. The cerebellum, through its role in articulation, is hypothesized to be essential for the development of phonological knowledge. But the phonological representations themselves, once established can be accessed without involving the cerehellum. A more direct relation is assumed by the proposal that dyslexia is a specific manifestation of a failure of skill automatization (Nicolson et al., 2001), assuming that such automatized skills entail the consolidation of representations within the cerebellum.

\section{THE CEREBELLUM AS AN INTERNAL TIMING SYSTEM}

We have sought to identify basic component operations that, in combination, might underlie a general human competence -- the ability to produce coordinated movements. Using an individual difference approach, we found that the ability to produce well-timed movements was highly correlated across different effectors like the finger and foot (Keele et al., 1985). In contrast, a much lower correlation was found between temporal control and response speed, as well as between temporal control and force control (Keele et al., 1987), even when the correlations involved performance with the same effector. The results of these studies suggest the existence of a specific system devoted to controlling the timing of movements, or what might be called an 'internal clock'. Further support for this hypothesis came from studies looking at correlations between these motor tasks and perceptual tasks. A significant correlation was found between motor and perceptual timing (Keele et al., 1985): Individuals 
who were good at controlling the timing of their movements also exhibited fine acuity in judging temporal differences between stimulus events. This ability was specific to the temporal domain; for example, motor timing and loudness perception were not correlated.

To examine the neural structures involved in internal timing, we tested various neurology patients on our tests of motor and perceptual timing. For the motor task, we used the repetitive tapping task introduced by Wing and Kristofferson (1973). In this task, the participant taps on a response key with the index finger, attempting to match the target rate set by an auditory metronome (for example, $550 \mathrm{~ms}$ ). After 10 responses, the metronome is terminated, and the task of the participant is to continue tapping at the same rate for another 30 responses. The analysis focuses on the standard deviation of the unpaced inter-tap intervals. This dependent variable serves as an indicator of the consistency of an internal timing system. Wing and Kristofferson had shown that the autocovariance of the time series of responses could be used to decompose this measure into two independent sources of variability. One source is associated with central processes determining when the next response should be produced, or what is referred to as 'clock variability' (but see Ivry \& Hazeltine, 1995); the other source is associated with response implementation, or what Wing termed 'motor delay'. In brief, the model assumes that an internal clock determines when each response is to be emitted, and this command must then be translated into a movement. Each process makes an independent contribution, resulting in the total variability of the interresponse intervals.

For the perceptual task, we used an adaptive psychophysical procedure to determine the difference threshold on a duration discrimination task. Four tones are presented on each trial, with the first two separated by a standard interval (for example, $400 \mathrm{~ms}$ ) and the second two separated by a variable interval. The participant judged whether the variable interval was shorter or longer than the standard interval. Based on this response, the duration of the variable interval is adjusted. For example, if the variable interval is longer than the standard yet the response was "shorter", then the variable interval for the next trial would be made longer. After a fixed number of trials, the duration of the variable interval provides an estimate of the difference required for performance at a predetermined criterion. A stable estimate of this difference threshold is obtained after about 30 trials. In this way, perceptual temporal acuity is measured; for example, a noisy internal clock would lead to a higher difference threshold. The same stimulus configuration is also used in a control task, but here the loudness of the second pair of tones is varied. This control task allows us to determine if someone has generic problems on perceptual tasks or whether the impairment is specific to one task or the other.

Three groups of patients were tested in our first study (Ivry \& Keele, 1989): (1) a group with either focal or degenerative cerebellar lesions, (2) a group with cortical lesions resulting in coordination problems, and (3) a group with Parkinson's disease. We assumed that the latter group was representative of basal ganglia dysfunction. The results showed that the integrity of the cerebellum was essential for accurate timing. The patients with cortical or cerebellar lesions were more variable on the tapping task, and this increase was primarily associated with the clock component (but see also Ivry et al., 1988). Moreover, only patients with cerebellar lesions were impaired on the duration discrimination task. Their difference threshold was about $50 \%$ greater than either of the other two patient groups and agematched controls. The deficit of these patients was 
specific to the time perception task; their performance was comparable to that of the controls on the loudness discrimination task. Interestingly, the Parkinson's patients were unimpaired on both the motor and the perceptual tasks (but see Harrington et al., 1998). This result was also found in a subset of patients tested both on and off their normal medication regimen. In the latter condition, their motor symptoms were exacerbated, yet remained comparable to controls on the timing tasks.

The results of these studies led us to postulate that the cerebellum plays a critical role in the precise representation of temporal information. As reviewed elsewhere (Ivry, 1997), this hypothesis is in accord with many of the prominent coordination problems associated with cerebellar dysfunction including intentional tremor, dysmetria, and speech dysarthria. The timing hypothesis also provides a computational account of the role of the cerebellum in certain types of sensorimotor learning like eyeblink conditioning. By this view, the cerebellum is essential for those tasks in which the learned response is adaptive only when the temporal relation between different environmental events must be extracted. In eyeblink conditioning, the animal must learn not only to anticipate an aversive stimulus like an air puff but also must learn exactly when that stimulus will occur so that the conditioned response is timed to maximally attenuate the aversive consequences of the air puff.

Subsequent research has supported the role of the cerebellum in temporal processing, although considerable debate continues about whether this structure is uniquely suited for this form of representation. Much remains to be learned at a mechanistic level. Nonetheless, the timing hypothesis provides a functional account of the cerebellar contribution to coordination and has offered novel insight into non-motor functions of this subcortical structure.

\section{ASSESSING CEREBELLAR FUNCTION IN CLUMSY CHILDREN}

As noted previously, it seems reasonable to ask if clumsiness is related, at least in part, to cerebellar dysfunction. First, the defining features of clumsiness are problems of coordination, the cardinal symptoms observed in patients with cerebellar ataxia. Second, the recent links between various developmental disorders and cerebellar abnormalities suggests that this structure may be especially vulnerable during early brain development. Nonetheless, few studies have focused on the question of whether clumsy children exhibit signs of cerebellar dysfunction. In this section, I review two studies that were published on this topic over a decade ago. Surprisingly, a literature search failed to reveal more recent papers that have pursued this issue, suggesting that a cognitive neuroscientific approach to the study of clumsiness has yet to be vigorously pursued.

The logic of our studies was quite simple -- do children diagnosed as clumsy show deficits on motor and perceptual timing tasks similar to those in adult patients with cerebellar lesions? In the first study (Williams et al., 1992), fifty children were recruited, based on referrals from their teachers concerning possible motor coordination problems. The children were given a short form of the Bruininks-Oseretsky Motor Proficiency Scale (Bruininks, 1978) and a clinical battery developed by one of the authors to assess perceptual-motor development problems (Williams, 1973). The children were categorized as clumsy if they were between the $40^{\text {th }}$ and $50^{\text {th }}$ percentile on the Bruininks-Oseretsky test and scored between 0.5 and 1.5 SD below normal on at least 6 of the 9 items in the clinical battery. The control group consisted of individuals who were at or above the $50^{\text {th }}$ percentile on the Bruininks-Oseretsky test and scored above $0.4 \mathrm{SD}$ below the mean on at least 6 
of the 9 items in the clinical battery. Thus, the definition of the groups was conservative. Severely uncoordinated children were excluded, and the children in the control group showed some motor problems based on teacher observation, yet failed to meet the clinical criterion for clumsiness. Based on these selection criteria, 12 children were assigned to the clumsy group and 13 to the control group.

Both groups were relatively proficient in maintaining the target interval during the unpaced phase of the tapping task, showing a slight hastening over the 30 taps. The clumsy children, however, exhibited greater overall variability, and when the data were analyzed with the Wing-Kristofferson model (Fig. 2a), only the estimate of clock variability was significant $(p<0.05$ vs. $p=0.61$ for the motor implementation estimate). The perception tasks also suggested a selective timing deficit (Fig. 2b). The mean difference threshold on the duration discrimination task was $54 \%$ higher for the group of clumsy children when compared with that of the control group ( $p<0.05)$. The two groups performed comparably on the loudness discrimination task $(p=0.66)$.

The results of Williams et al. (1992) show that children classified as clumsy on standard clinical assessment instruments are impaired on tasks that require precise timing. We hypothesize that their deficits on the two timing tasks reflect cerebellar dysfunction, given the similarity of their performance profiles to that exhibited by adult patients with acquired cerebellar lesions. We cannot, of course, claim on the basis of these results that cerebellar dysfunction is directly causal for the clumsiness of these children. Indeed, as with single dissociations in neuropsychology, the results are of limited value in evaluating the specificity of the neural correlates of clumsiness. The normal performance of the clumsy children on the loudness task demonstrates that this group does not perform poorly on all tasks: on the task employed, their impairments are restricted to those
A.

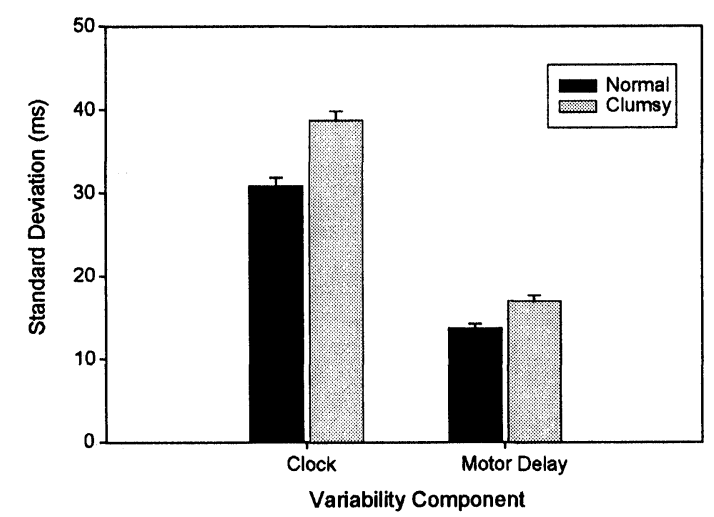

B.

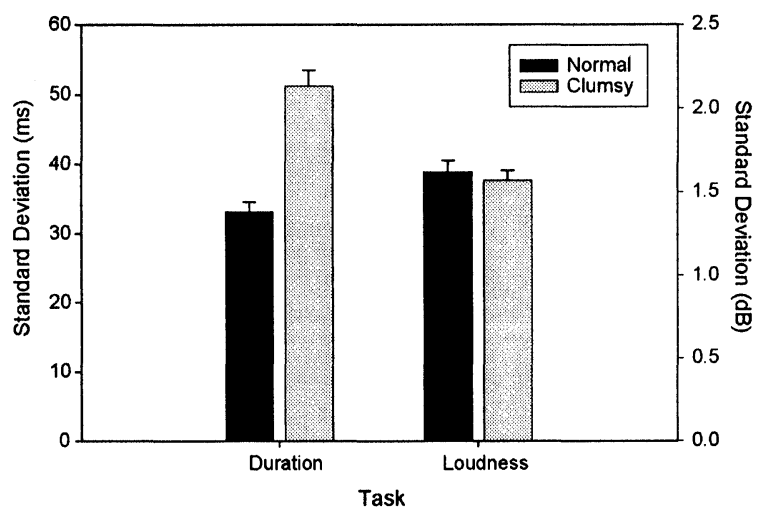

Fig. 2: Performance of normal and clumsy children on motor and perceptual timing tasks used to assess cerebellar function. a) Estimates of component sources of variability during repetitive finger tapping. The total variability of the inter-tap intervals during the unpaced phase of the trial is assumed to reflect independent contributions from central processes (CLOCK) determining when a response should be initiated and motor implementation processes (MOTOR DELAY), involved in executing the central commands. b) Difference thresholds on two perceptual discrimination tasks, duration (left axis) and loudness (right axis). The values indicate the difference required between the standard and comparison stimuli for the participants to be correct on $75 \%$ of the trials. Adapted from Williams et al. (1992). 
that evaluate the operation of an internal timing system. But it may well be that these children would also be impaired on tasks that assess other components of coordination, not just timing. There may be a general deficit in the function of the entire motor system, with the (cerebellar) timing problems just one particular manifestation of this generic impairment

As noted by many researchers, the term 'clumsiness' is applied to a heterogeneous population. This practice raises the possibility that cerebellar dysfunction might be present in one subgroup of clumsy children and absent in other subgroups. Such a result would suggest that developmental movement disorders might mirror those in patients with acquired neurologic lesions. Similar to how these acquired lesions produce system-specific impairments, subtypes of clumsiness might reflect the dysfunctional operation of limited sets of neural systems. Alternatively, the cluster of symptoms that define clumsiness might arise only when there is widespread depression of neural function or may not pinpoint specific neural abnormalities with such heterogeneity.

Laurie Lundy-Ekman addressed this specificity question in her dissertation studies (Lundy-Ekman et al., 1991). The design of her study was similar to that of Williams et al. (1992). The selection procedure, however, was modified to include a neurologic exam that was created to assess the presence of soft neurologic signs of basal ganglia or cerebellar dysfunction (Touwen, 1979). The term 'soft signs' is used when evidence of a neurologic disorder is absent (for example, reflex abnormality or known brain injury), yet when given a neurologic exam, the performance is similar to that seen in patients with known neurologic disorders. For basal ganglia function, the assessment was for signs associated with Huntington's disease. Such signs included testing for choreiform and athetoid movements, or the presence of synkinesis (for example, when children spread their fingers with arms outstretched, small jerky movements were rated as choreiform, those with slow writhing movements were rated as athetoid). Cerebellar function was assessed by tests for dysdiadokinesis (for example, smoothness of repetitive wrist pronation and supination), intentional tremor, and dysmetria.

A total of 155 7- and 8-year olds were given the neurologic exam. Of these, 60 exhibited soft neurologic signs. Twenty were excluded because they presented both soft basal ganglia and soft cerebellar signs. The others were given the Bruininks-Oseretsky test and selected for the study if they scored below the $40^{\text {th }}$ percentile. The final groups consisted of 11 children with soft signs of basal ganglia dysfunction and 14 children with soft signs of cerebellar dysfunction. Fourteen control participants were selected from the pool of candidates who did not present any soft neurologic signs and scored above the $40^{\text {th }}$ percentile on the Bruininks-Oseretsky test (mean of $79^{\text {th }}$ percentile).

The task battery included the tapping task, the duration and loudness discrimination tasks, and a force control task. The latter was chosen because patients with Parkinson's disease are impaired in the ability to modulate force output (Hallett \& Khoshbin, 1980; Ivry \& Corcos, 1993; Wing, 1988). For this task, isometric movements were made with the index finger on a strain gauge. Target force levels were indicated by the vertical position of a line appearing on the computer monitor (for example, for a large target force, the line was positioned near the top of the screen). The same target was used for 12 consecutive responses. Feedback was provided for the first six responses. No feedback was given for the last six. As with the tapping task, the focus was on the consistency (standard deviation) with which the participants produced a series of responses without feedback.

The results revealed a striking dissociation between the performances of children with soft cerebellar or soft basal ganglia signs. In terms of 
total variability on the tapping task, children with soft cerebellar signs were significantly more variable than both the controls and children with soft basal ganglia signs (Fig. 3a). When the data were analyzed with the Wing-Krisotofferson model, the only reliable difference was between the controls and the soft cerebellar group in estimates of clock variability $(p<0.05)$. A similar dissociation was also observed in the perception tasks (Fig. 3b). The difference in the threshold on the duration discrimination task was much larger for the children with soft cerebellar signs than for the other two groups $(p<0.05)$. No differences were observed on the control loudness discrimination task. A very different picture emerged on the force control task. Here, the children with basal ganglia soft signs were more variable than were the other two groups (Fig. 3c). Note that the children with soft cerebellar signs tended to produce smaller forces than did the other two groups.
A.

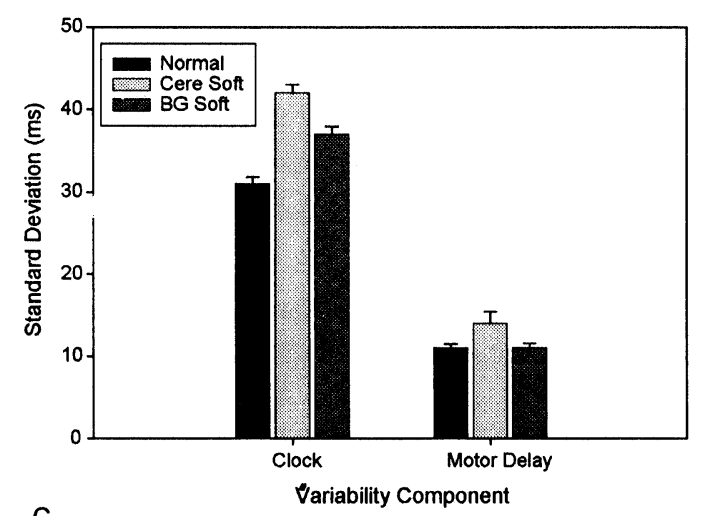

C.

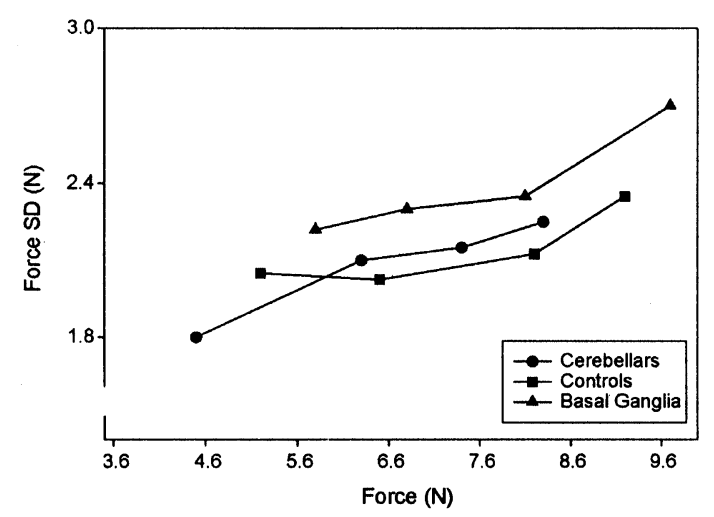

B.

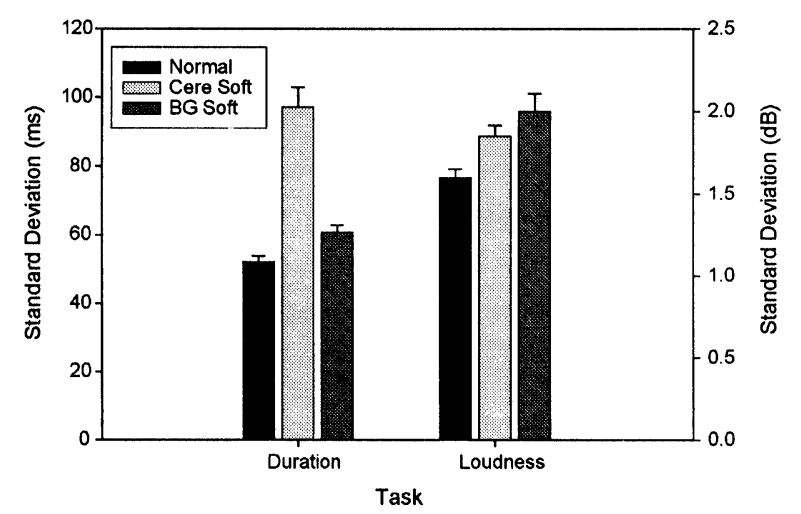

Fig. 3: Performance of normal and clumsy children on motor and perceptual tasks used to assess subcortical function. Based on an independent clinical exam, the clumsy children were divided into two groups, those with soft signs of cerebellar dysfunction and those with soft signs of basal ganglia dysfunction. (a) As in Figure 2, estimates of component sources of variability during repetitive finger tapping, and (b) difference thresholds on two perceptual discrimination tasks. (c) Variability of force pulses produced without feedback is plotted as a function of mean force produced. Adapted from Lundy-Ekman et al. (1991). 
When a normalized measure of variability was used (SD divided by mean force), the group difference was only marginally significant. Even with this adjustment, however, the normalized measure of variability remained highest for the children with soft basal ganglia signs $(p<0.05)$.

In suns nary, the results of the Lundy-Ekman study (1991) suggest that some degree of specificity, in terms of the underlying neurologic dysfunction, may be associated with clumsiness, at least for subpopulations of children. A group of clumsy children was identified who presented soft neurologic signs of cerebellar dysfunction. Similar to adults with acquired cerebellar lesions, these children were selectively impaired on tasks that required precise timing. In contrast, the children with soft basal ganglia signs performed normally on the tapping and duration discrimination tasks. The soft basal ganglia group, however, was impaired on the force control task. Thus, their motor problems, in terms of both clinical assessmert and behavioral performance, are similar to that found in patients with basal ganglia dysfunction.

As interesting as the patterns of impairment are, noteworthy is that the clumsy children did not perform more poorly than the controls on all motor tasks. The basal ganglia group was unimpaired on the tapping task, and the performance of the cerebellar group, at least on measures of variability, was similar to that of the control group on the force control task. The results argue against the hypothesis that clumsiness reflects a generalized dysfunction across the motor system. For at least some children, the syndrome may reflect dysfunction in a particular neural system. Of course for others, the problems can be more widespreaa, as indicated by the significant percentage of children exhibiting both soft cerebellar and soft basal ganglia signs. We would predict that these children would have performed poorly on both the timing and force control tasks.

\section{CONCLUSIONS}

The studies reviewed in this paper provide an example of the bidirectional nature of cognitive neuroscience research. At the behavioral level, a primary endeavor within the field is to specify the computations that allow for different aspects of mental competence. Tasks used in clinical assessments prove to have good utility for discriminating between normal and abnormal populations, but the complexity of many of these tasks, limits their utility for evaluating specific functional hypotheses. The focus of cognitive neuroscience research is on the computational level. The finding that clumsy children are more variable than age-matched controls in producing regularly timed intervals is not surprising. The conclusion, however, that some of these children have a problem representing temporal information is bolstered by the observation of a corresponding impairment on a perceptual timing task. As important, the finding that other children, rated as equally clumsy on standard clinical assessment batteries, are not impaired on the timing tasks provides stronger evidence that a timing problem may be present in a subpopulation of clumsy children.

At the neural level, it remains to be seen if developmental disorders like clumsiness reflect abnormal function in a single or a limited set of neural structures or whether they result from diffuse abnormalities. Given that the clinical picture is heterogeneous, it is likely that a multitude of neurologic profiles are also associated with clumsiness. The results of the study of LundyEkman et al. (1991) demonstrate that distinct subpopulations of clumsy children have behavioral problems similar to those of patients with either cerebellar or basal ganglia dysfunction. Thus, for these subgroups, there may be some neural specificity. On the other hand, the story that has emerged in the search for the neural basis of autism 
may be instructive here. In the initial highresolution MRI studies, the only region showing structural abnormalities was the cerebellum. This finding led to considerable effort to determine how cerebellar dysfunction would cause autism. Subsequent studies, however, have shown that neural abnormalities are quite widespread in autism, with reduced volume reported in limbic, cortical, and white matter regions (Courchesne, 1997). Although a link between cerebellar dysfunction and autism might still exist, a simple mapping between neural pathology and behavioral syndrome seems very unlikely.

\section{REFERENCES}

Altman J, Bayer SA. 1985. Embryonic development of the rat cerebellum. III. Regional differences in the time of origin, migration, and settling of Purkinje cells. J Comp Neurol 23 1: 42-65.

Andreasen NC, O'Leary DS, Cizadlo T, Arndt S, Rezai K, Ponto LL, et al. 1996. Schizophrenia and cognitive dysmetria: a positron-emission tomography study of dysfunctional prefrontalthalamic-cerebellar circuitry 1996 Proc Natl Acad Sci USA 93: 9985-9990.

Berquin PC, Giedd JN, Jacobsen LK, Hamburger SD, Krain AL, Rapoport JL, et al. 1998. Cerebellum in attention-deficit hyperactivity disorder: a morphometric MRI study. Neurology 50: 1087-1093.

Bruininks RH. 1978. Bruininks-Oseretzky test of motor proficiency, Examiner's manual. Circle Pines, Minnesota, USA: American Guidance Service.

Coltheart M, Rastle K, Perry C, Langdon R, Ziegler J. 2001. DRC: a dual route cascaded model of visual word recognition and reading aloud. Psychol Rev 108: 204-256.

Courchesne E, Yeung-Courchesne R, Press GA, Hesselink JR. 1988. Hypoplasia of cerebellar vermal lobules VI and VII in autism. New Engl J Med 318: 1349-1354.

Courchesne E, Townsend J, Saitoh O. 1999. The brain in infantile autism: Posterior fossa structures are abnormal. Neurology 44: 214-223.

Courchesne E. 1997. Brainstem, cerebellar and limbic neuroanatomic abnormalities in autism. Curr Opin Neurobiol 7: 269-278.
Courchesne E, Allen G. 1997. Prediction and preparation, fundamental functions of the cerebellum. Learn Mem 4: 1-35.

Demb JB, Boynton GM, Heeger DJ. 1998. Functional magnetic resonance imaging of early visual pathways in dyslexia. J Neurosci 18: 6939-6951.

Fawcett AJ, Nicolson RI, Dean P. 1996. Impaired performance of children with dyslexia on a range of cerebellar tasks. Ann Dyslexia 46: 259-283.

Fawcett AJ, Nicolson RI. 1999. Performance of dyslexic children on cerebellar and cognitive tests. J Mot Behav 31: 68-78.

Hallett M, Khoshbin S. 1980. A physiological mechanism of bradykinesia. Brain 103: 301-314.

Harrington DL, Haaland KY, Hermanowitz N. 1998. Temporal processing in the basal ganglia. Neuropsychology 12: 3-12.

Helmuth LL, Ivry RB, Shimizu N. 1997. Preserved performance by cerebellar patients on tests of word generation, discrimination learning, and attention. Learn Mem 3: 456-474.

Ivry R. Cerebellar timing systems. 1997. Int Rev Neurobiol 41: 555-573.

Ivry RB, Keele SW, Diener HC. 1988. Dissociation of the lateral and medial cerebellum in movement timing and movement execution. Exp Brain Res 73: $167-180$.

Ivry RB, Keele SW. 1989. Timing functions of the cerebellum. J Cognit Neurosci 1: 136-152.

Ivry RB, Corcos D. 1993. Slicing the variability pie: Component analysis of coordination and motor dysfunction. In: Newell $\mathrm{K}$, Corcos $\mathrm{D}$, eds, Variability and Motor Control. Champaign, Illinois, USA: Human Kinetics Publishers; 415-447.

Ivry RB, Hazeltine RE. 1995. Perception and production of temporal intervals across a range of durations: Evidence for a common timing mechanism. J Exp Psychol: Hum Percept Perform 21: 3-18.

Ivry RB, Justus TC, Middleton C. 2001. The cerebellum, timing, and language: Implications for the study of dyslexia. In: Wolf $\mathrm{M}$, ed, Dyslexia, Fluency, and the Brain. Timonium, Maryland, USA: York Press; 189-211.

Keele SW, Pokorny RA, Corcos DM, Ivry R. 1985. Do perception and motor production share common timing mechanisms: A correlational analysis. Acta Psychol 60: 173-191.

Keele SW, Ivry RI, Pokorny RA. 1987. Force control and its relation to timing. J Mot Behav 19: 96-114. 
Lundy-Ekman L, Ivry RB, Keele S, Woollacott M. 1991. Timing and force control deficits in clumsy children. J Cognit Neurosci 3: 367-376.

Mercuri E, Barnett AL. 2003. Motor outcome at school age in children with neonatal encephalopathy: Correlation with neonatal brain MRI. Neural Plast 10: 51-58-this issue.

Mostofsky SH, Reiss AL, Lockhart P, Denckla MB. 1998. Evaluation of cerebellar size in attentiondeficit hyperactivity disorder. J Child Neurol 13: 434-439.

Nicolson RI, Fawcett AJ, Dean P. 2001. Developmental dyslexia: The cerebellar deficit hypothesis. Trends Neurosci 24: 508-511.

Nopoulos PC, Ceilley JW, Gailis EA, Andreasen NC. 1999. An MRI study of cerebellar vermis morphology in patients with schizophrenia: evidence in support of the cognitive dysmetria concept. Biol Psychiatry 46: 703-711.

Schmahmann J, Harris R, editors. 1997. The Cerebellum and Cognition. San Diego, California, USA: Academic Press Inc.; 665.

Studdert-Kennedy M, Mody M. 1995. Auditory temporal perception deficits in the readingimpaired: A critical review of the evidence. Psychon Bull Rev 2: 508-514.
Touwen BCL. 1979. The neurologic examination of the child with minor nervous dysfunction. $2^{\text {nd }}$ edition. London, England: William Heinemann Medical Books; 105.

Townsend J, Courchesne E, Covington J, Westerfield M, Harris NS, Lyden P, et al. 1999. Spatial attention deficits in patients with acquired or developmental cerebellar abnormality. J Neurosci 19: 5632-5643.

Williams HG. 1973. The Williams Gross Motor Control Test Battery. Toledo, Ohio, USA: University of Toledo.

Williams HG, Woollacott MH, Ivry R. 1992. Timing and motor control in clumsy children. $\mathrm{J}$ Mot Behav 24: 165-172.

Wing A, Kristofferson A. 1973. Response delays and the timing of discrete motor responses. Percept Psychophys 14: 5-12.

Wing AM. 1988. A comparison of the rate of pinch grip force increases and decreases in Parkinsonian bradykinesia. Neuropsychologia 26: 479-482.

Wiser AK, Andreasen NC, O'Leary DS, Watkins GL, Ponto LLB, Hichwa RD. 1998. Dysfunctional cortico-cerebellar circuits cause 'cognitive dysmetria' in schizophrenia. Neuroreport 8: 1895 1899. 

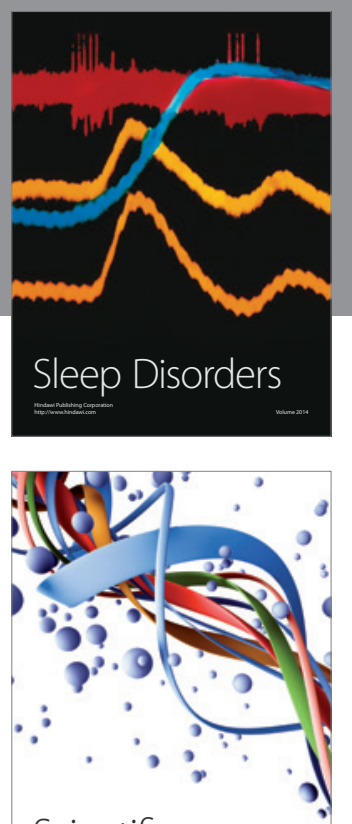

Scientifica
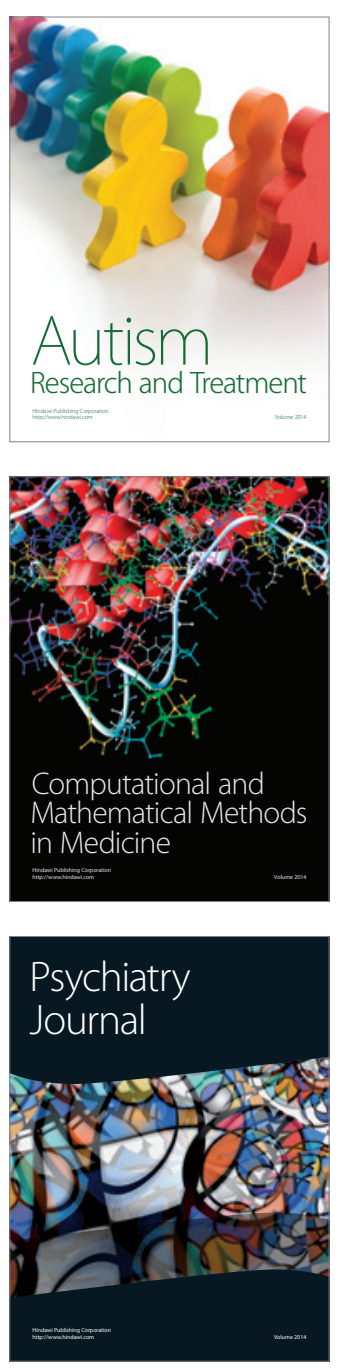
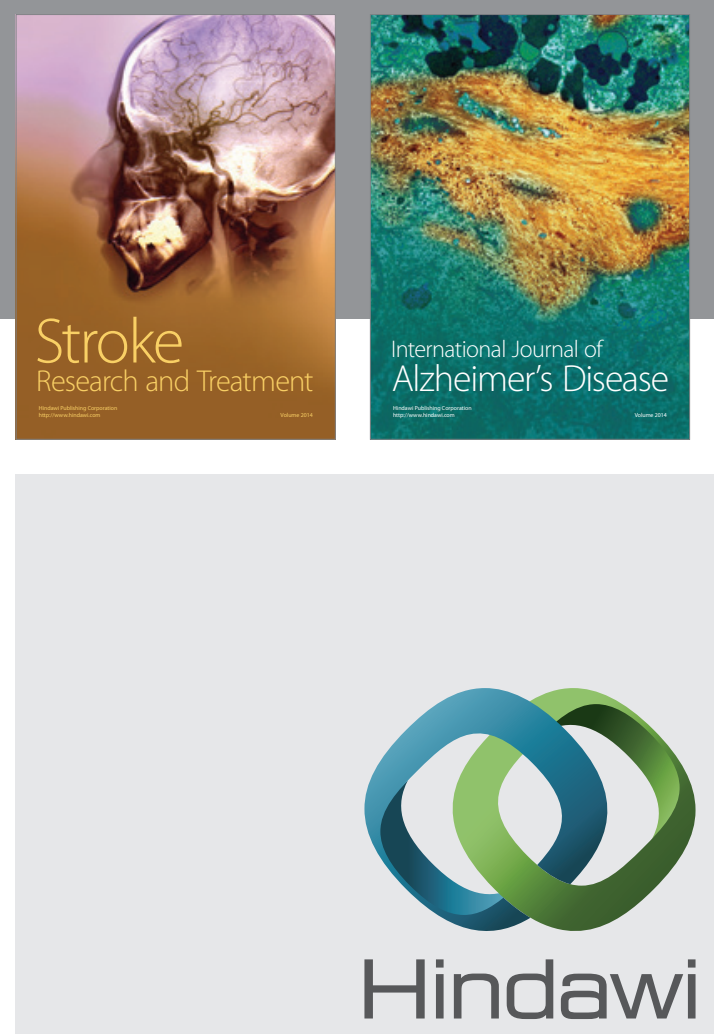

Submit your manuscripts at

http://www.hindawi.com
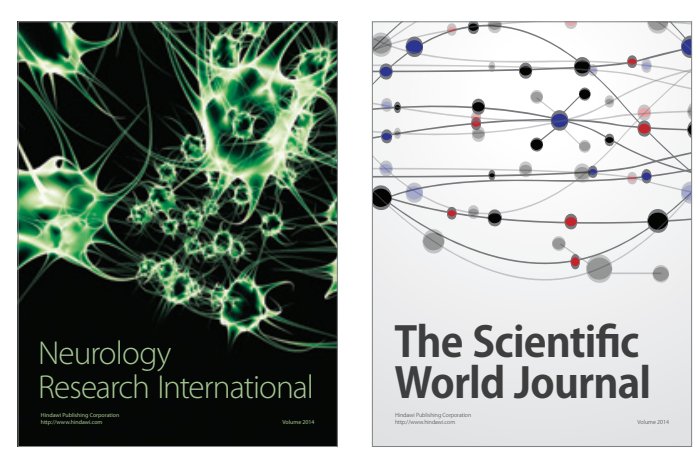

The Scientific World Journal

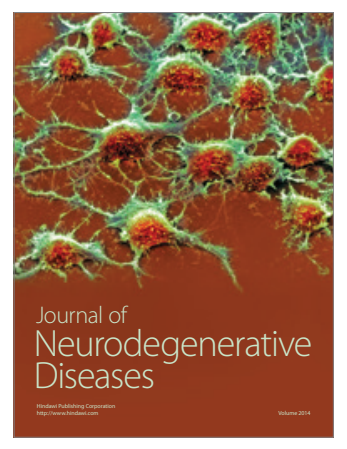

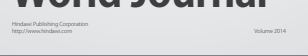

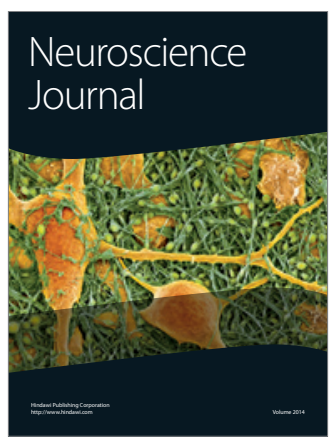

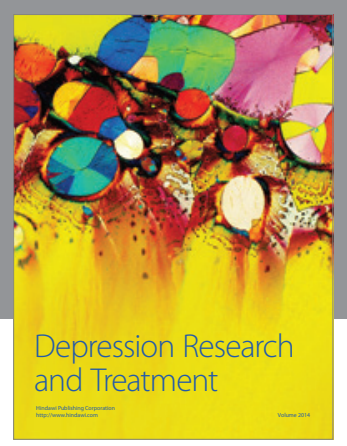
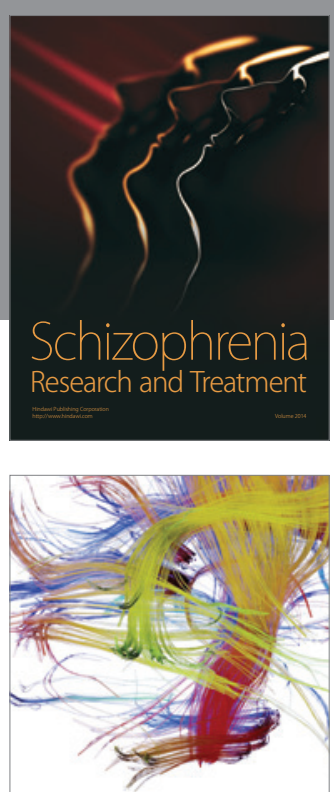

Brain Science

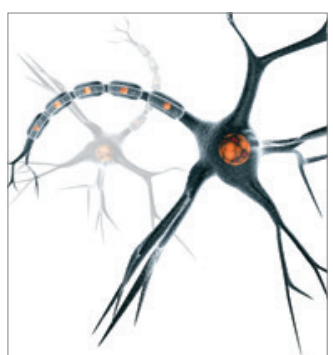

Neural Plasticity
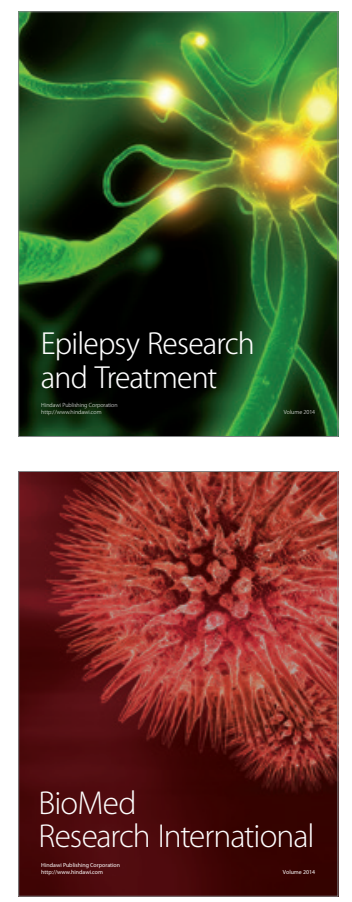

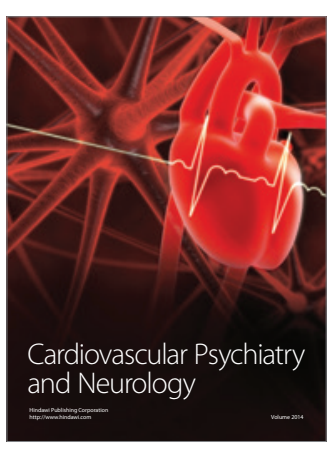

Parkinson's

Disease
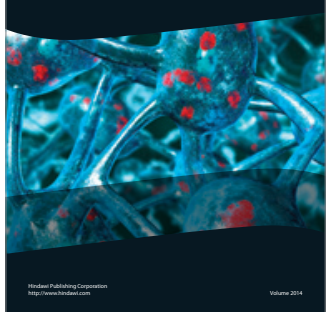